\title{
Characterisation of children's head motion for Magnetic Resonance Imaging with and without general anaesthesia
}

\author{
Hannah Susanne Eichhorn ${ }^{\mathrm{a}, \mathrm{b}}$, Andreea-Veronica Vascan ${ }^{\mathrm{a}, \mathrm{c}}$, Martin \\ Nørgaard ${ }^{\mathrm{a}, \mathrm{d}}$, Andreas Høyby Ellegaard ${ }^{\mathrm{e}}$, Jakob Slipsager ${ }^{\mathrm{f}, \mathrm{g}, \mathrm{e}}$, Sune Høgild \\ Keller $^{\mathrm{g}}$, Lisbeth Marner ${ }^{\mathrm{g}, \mathrm{h}}$, Melanie Ganz ${ }^{\mathrm{a}, \mathrm{c}, *}$ \\ ${ }^{a}$ Neurobiology Research Unit, Copenhagen University Hospital, Rigshospitalet, Copenhagen, \\ Denmark \\ ${ }^{b}$ Niels Bohr Institute, University of Copenhagen, Copenhagen, Denmark \\ ${ }^{c}$ Department of Computer Science, University of Copenhagen, Copenhagen, Denmark \\ ${ }^{d}$ Center for Reproducible Neuroscience, Department of Psychology, Stanford University, \\ Stanford, CA, USA \\ e TracInnovations, Ballerup, Denmark \\ ${ }^{f}$ DTU Compute, Technical University of Denmark, Lyngby, Denmark \\ ${ }^{g}$ Department of Clinical Physiology, Nuclear Medicine \& PET, Copenhagen University \\ Hospital Rigshospitalet, Copenhagen, Denmark \\ ${ }^{h}$ University Hospital Bispebjerg, Copenhagen, Denmark.
}

\begin{abstract}
Head motion is one of the major reasons for artefacts in Magnetic Resonance Imaging (MRI), which is especially challenging for children who are often intimidated by the dimensions of the MR scanner. In order to optimise the MRI acquisition for children in the clinical setting, insights into children's motion patterns are essential. In this work, we analyse motion data from 61 pediatric patients. We compare structural MRI data of children imaged with and without general anaesthesia (GA), all scanned using the same hybrid PET/MR scanner. We analyse several metrics of motion based on the displacement relative to a reference, decompose the transformation matrix into translation and rotation, as well as investigate how different regions in the brain are affected by motion. Head motion for children without GA was significantly higher (mean displacement of $2.19 \pm 0.93 \mathrm{~mm}$ (median \pm standard deviation) during $41.7 \pm 7.5 \mathrm{~min}$
\end{abstract}

\footnotetext{
* Corresponding author

Email address: melanie.ganz@nru.dk (Melanie Ganz)
} 
scans); however, even anaesthetised children showed substantial residual head motion (mean displacement of $1.12 \pm 0.35 \mathrm{~mm}$ ). For both patient groups translation along the z-axis (along the scanner bore) was significantly larger in absolute terms (GA / no GA: $0.87 \pm 0.29 \mathrm{~mm} / 0.92 \pm 0.49 \mathrm{~mm}$ ) compared to the other directions. Considering directionality, both patient groups were moving in negative z-direction and thus, out of the scanner. The awake children additionally showed significantly more nodding rotation $\left(0.33 \pm 0.20^{\circ}\right)$. Consequently, in future studies as well as in the clinical setting, these predominant types of motion need to be taken into consideration to limit artefacts and reduce re-scans due to poor image quality.

Keywords: Magnetic Resonance Imaging, Motion patterns, Motion Artefact, Children, Brain

\section{Introduction}

For Magnetic Resonance Imaging (MRI), artefacts are most frequently caused by patient head motion due to long acquisition times of typically 30 to 60 minutes [1]. These artefacts manifest as ghosting, blurring or signal variations, thus

5 reducing overall image quality and resulting in unsuccessful diagnoses [2]. Andre et.al. 3] determined the percentage of at least partly repeated MRI examinations to approximately $20 \%$, leading to an estimated increased cost of 115,000 US dollars per scanner per year in the US due to motion.

Children tend to move more in the MR scanner than adults since they are more negatively influenced by the large size of the scanner, the narrow bore and the loud noises during image acquisition [4]. So far, motion artefacts for children between 4 and 10 years are mostly reduced by sedation or general anaesthesia (GA) [5], with the exact age limit being hospital specific. However, the need for an anaesthetist to administer the drug and monitor the child, increases the 15 patient's waiting times, as well as the costs of the examination. Slipsager et.al. [6] calculated the additional cost for using GA in MRI examinations to 319,000 US dollars per scanner per year in Denmark. Furthermore, concerns about 
adverse events like airway obstruction or oxygen desaturation of GA in young children are increasing $[1,4,7,8$.

Currently, different approaches for preventing motion artefacts without general anaesthesia or sedation are under research. These include strategies for preparing the children with story books and mock scanners before they undergo MRI and distracting them visually and acoustically during the examination [4, 5. 9, 10. Another approach is to apply motion correction during or after image acquisition, for which a variety of methods have been developed and tested. These include prospective motion correction updating the position of the field of view in real time dependent on motion estimates [11, 12, 13], as well as several retrospective techniques using mathematical properties of the Fourier Transform, Compressed Sensing or Machine Learning [14, 15, 16, 17].

For both strategies - preparation and motion correction - information about the children's motion patterns in the MR scanner is essential. In the case of motion correction algorithms, additional information is needed for tailoring them towards children specific movements. For the preparation strategy as well as in the clinical setting, information about predominant motion patterns is critical for preventing those types of motion. Churchill et.al. [18 analysed head motion of adults during fMRI using retrospective image-based motion correction. Overall, they found low motion estimates with only two cases of estimates above $1 \mathrm{~mm}$ or $1^{\circ}$ for the whole brain. The largest standard deviation of the displacement relative to a reference image was observed for pitch movement 40 (nodding). Afacan et.al. [1] investigated children's head motion and its impact on image quality. Their analysis showed a correlation of mean displacement and motion free time to image quality, but no statistically significant correlation of maximum displacement. Additionally, they did not find a significant correlation between the analysed motion metrics and age. Together, these results suggest 45 that head motion is a challenge, not only for young and uncooperative children.

The aim of this work is to analyse motion patterns of children in order to draw possible conclusions about predominant movement habits and thus, enable optimisation of motion correction methods for specific types of motion. For that, 


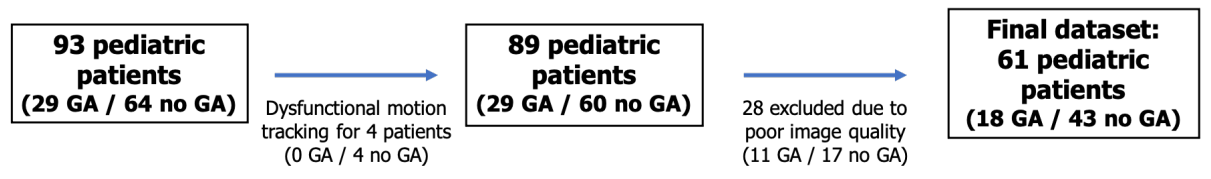

Figure 1: Schematic overview of exclusion process.

\begin{tabular}{l|c|c|c}
\multicolumn{4}{|c}{ Table 1: Demographic information for patient population } \\
& GA & No GA & All \\
\hline Number of patients (sex) & $18(10$ male $)$ & $43(28$ male $)$ & $61(38$ male $)$ \\
Mean age \pm std & $5.94 \pm 2.75$ & $12.65 \pm 3.04$ & $10.67 \pm 4.25$ \\
Age range & $21 \mathrm{~m}-13 \mathrm{y}$ & $7 \mathrm{y}-19 \mathrm{y}$ & $21 \mathrm{~m}-19 \mathrm{y}$
\end{tabular}

we also investigate how head motion translates into motion of different parts of the brain. The analyses will be performed both for children imaged with and without GA.

\section{Material and Methods}

\subsection{Patient population}

Within this study, MR and motion data from 93 pediatric patients with brain tumors acquired in a previous study were analysed [19]. As visualised in Figure 1 four of those data sets were excluded due to dysfunctional motion tracking. Twenty eight additional data sets were excluded due to poor scan quality (ringing or blurring), large tumors or removal of large parts of the brain. Examples of excluded scans as well as a comparison of motion between excluded

${ }_{60}$ and included patients are provided in the Supplementary Material S.1 and S.2. Consequently, the final dataset consists of 61 patients aged between 21 months and 19 years, out of which 18 were scanned with GA (propofol and sevoflurane) and 43 were scanned without. Additional demographic information is provided in Table 1. Please note the larger age range of anaesthetised children, which is 65 due to clinical considerations. 
Table 2: MR Sequences

\begin{tabular}{c|c} 
Sequence & Scan Duration $[\mathbf{s}]$ \\
\hline $\mathrm{T}_{1}$-weighted MPRAGE & 266 \\
$\mathrm{~T}_{1}$-weighted STIR & 174 \\
$\mathrm{~T}_{2}$-weighted FLAIR (transversal) & 272 \\
$\mathrm{~T}_{2}$-weighted FLAIR (coronal) & 164 \\
$\mathrm{~T}_{2}$-weighted Blade & 122
\end{tabular}

The previous study, in which our data was aquired, was approved by the Danish National Committee on Health Research Ethics (approval H-6-2014-095) and was registered at clinicaltrials.gov (NCT03402425). Written informed consent was obtained from all patients / parents of the patients.

\subsection{MRI acquisition and region segmentation}

MR scans were performed using the mMR Biograph hybrid PET/MRI scanner (Siemens Healthineers, Erlangen, Germany) between April 2015 and January 2019, using a PET/MR child brain tumor protocol. The median wall time from the first to the last MR sequence was $41.7 \pm 7.5$ minutes. The MRI sequences of this protocol are listed in Table 2. Parameters of these sequences are reported in the Supplementary Material (Table S.1. The quality of the majority of the 61 scans in the final dataset was assessed as "optimal for clinical use" by radiologists, with only $6.3 \%$ of the scans scored "useful for diagnosis, but not optimal", as previously described by Slipsager et.al. [6].

The 3D-encoded, $\mathrm{T}_{1}$-weighted MPRAGE scans were processed with FreeSurfer 20] in order to segment 8 cortical and 8 subcortical regions: left and right hippocampus, caudate, amygdala, putamen, lateral occipital, inferior temporal, precentral and medial orbitofrontal regions. The centroids of these regions are visualised in Figure 2 . 


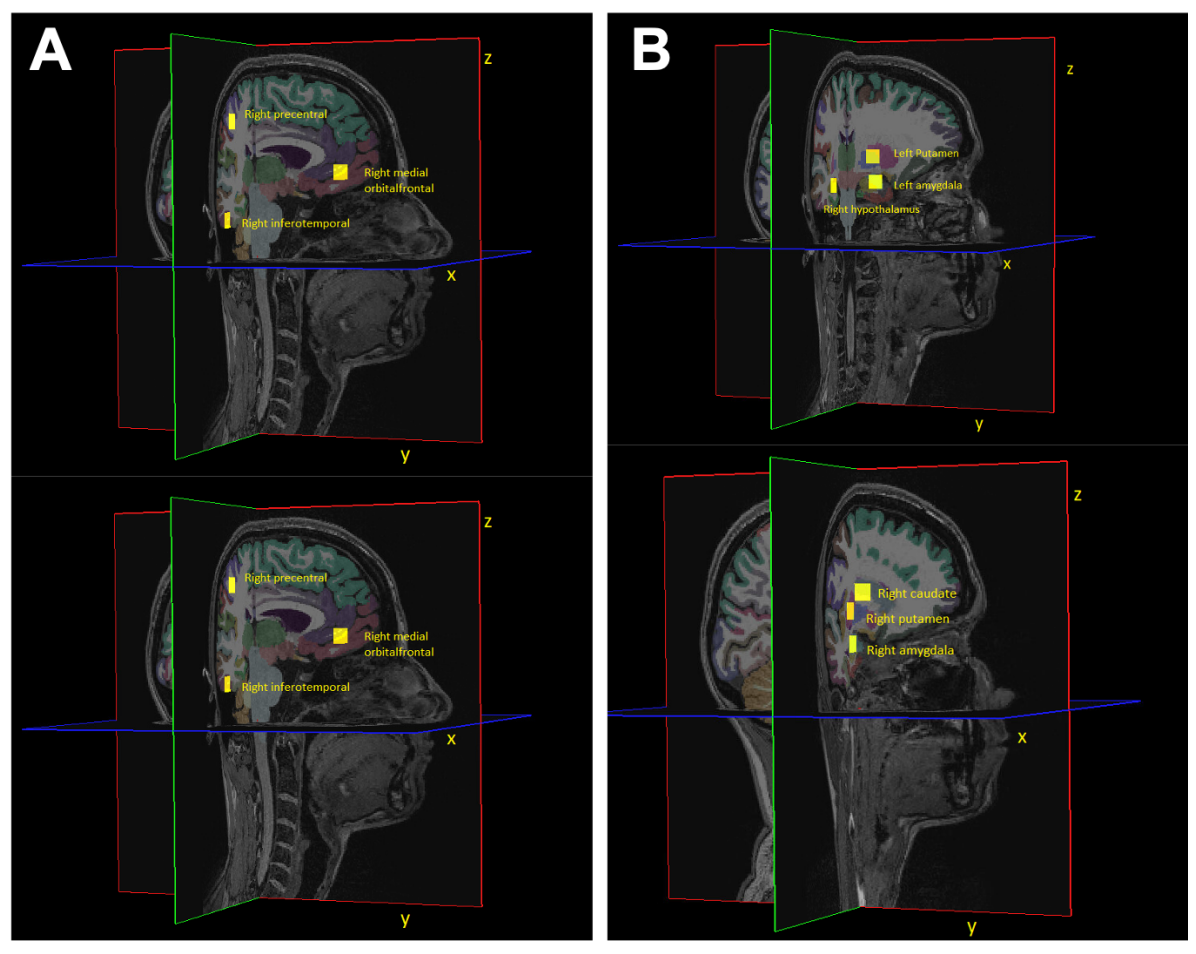

Figure 2: Visualisation of (A) 8 cortical and (B) 8 subcortical regions together with their centroid (yellow square). 
The patient's head motion was estimated with the markerless tracking system Tracoline (TracInnovations, Ballerup, Denmark) [21, 22]. It transmits non-visible infrared light onto the patient's face and estimates a 3D point cloud surface, the position of which is continuously measured at a frequency of approximately $30 \mathrm{~Hz}$. An example of this point cloud for an adult as well as a schematic visualisation of the point cloud centroid is shown in Figure S.3 of the Supplementary Material. Our analysis in the following was only performed on the motion data sampled during acquisition of the sequences summarized in Table 2. For each motion estimate, the middle time point of the MPRAGE acquisition was chosen as the reference position, since this time point corresponds to the acquisition of the center of k-space. Using a calibration transform, the estimates were moved into the RAS (Right, Anterior, Superior) coordinate system.

\subsection{Motion quantification}

Motion Metrics. In line with the analysis of Afacan et al. [1, the displacement of the point cloud centroid to the reference position was calculated for all time points as Euclidean distance to the reference position. For each patient, the mean, median and maximum of the displacement was determined. Additionally, motion-free time was quantified as the percentage of time, where the displacement relative to the reference position was below $2 \mathrm{~mm}$. This threshold was chosen different from Afacan et al. [1], since $2 \mathrm{~mm}$ is a standard value for motion thresholding at our institute.

Analogously, for each region the displacement of the region's centroid (cf. Figure 2 and the corresponding metrics were computed.

Matrix decomposition. Tracoline provides an estimate of the rigid body transformation matrix at each time point. Analogous to Churchill et al. [18, we decomposed each transformation matrix into translational and rotational components, which reveal the translation $(\mathrm{mm})$ along and rotation (degrees) around the three axes. 


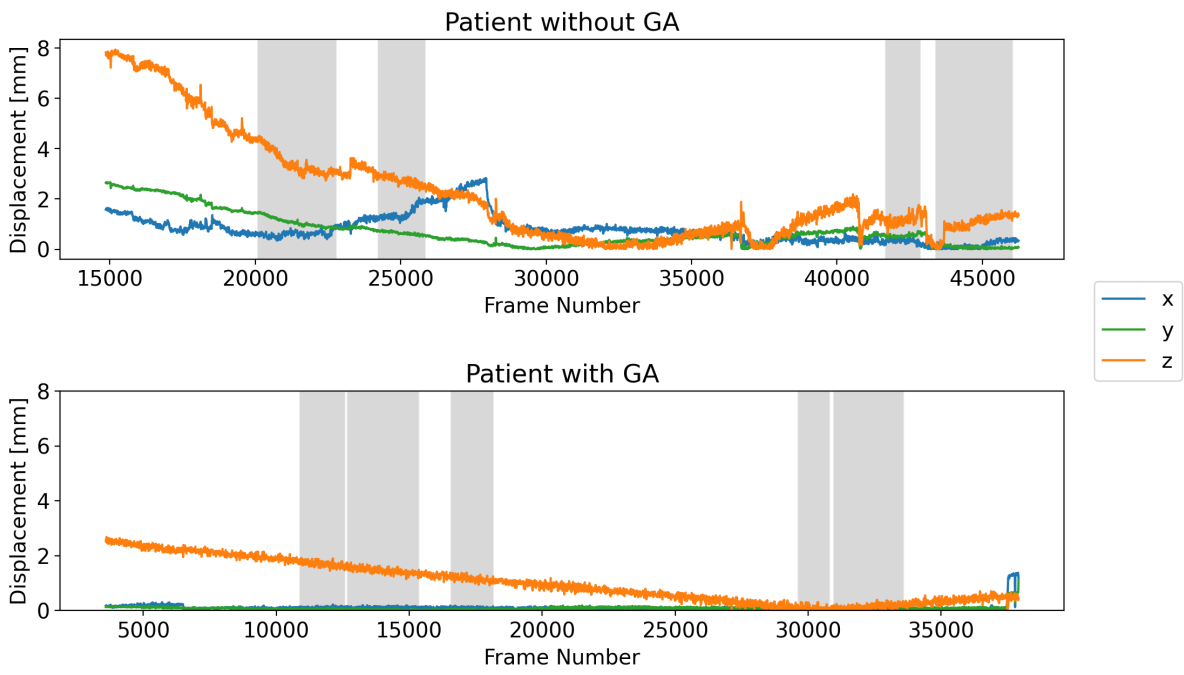

Figure 3: Example of motion curves for children with and without GA. Grey background inidcates the times where MR sequences have been acquired.

\subsection{Statistical analysis}

Statistical differences in motion metrics as well as in absolute translational and rotational components between groups and across brain regions $(\mathrm{N}=16)$ were evaluated using the Mann-Whitney U test. Correction for multiple comparisons across regions was carried out using False-Discovery Rate [23] (FDR), at $\mathrm{FDR}=0.05$.

\section{Results}

\subsection{Exemplary motion curves}

Exemplary motion curves during the entire scan session are shown in Figure 3 for one patient with and one without GA. Curves for other GA patients are similar to the shown example, whereas the curves for awake children show more variation.

\subsection{Comparison of motion metrics}

Figure 4 compares mean, median and maximum displacement, as well as motion free time for patients with and without GA. All metrics show a statis- 

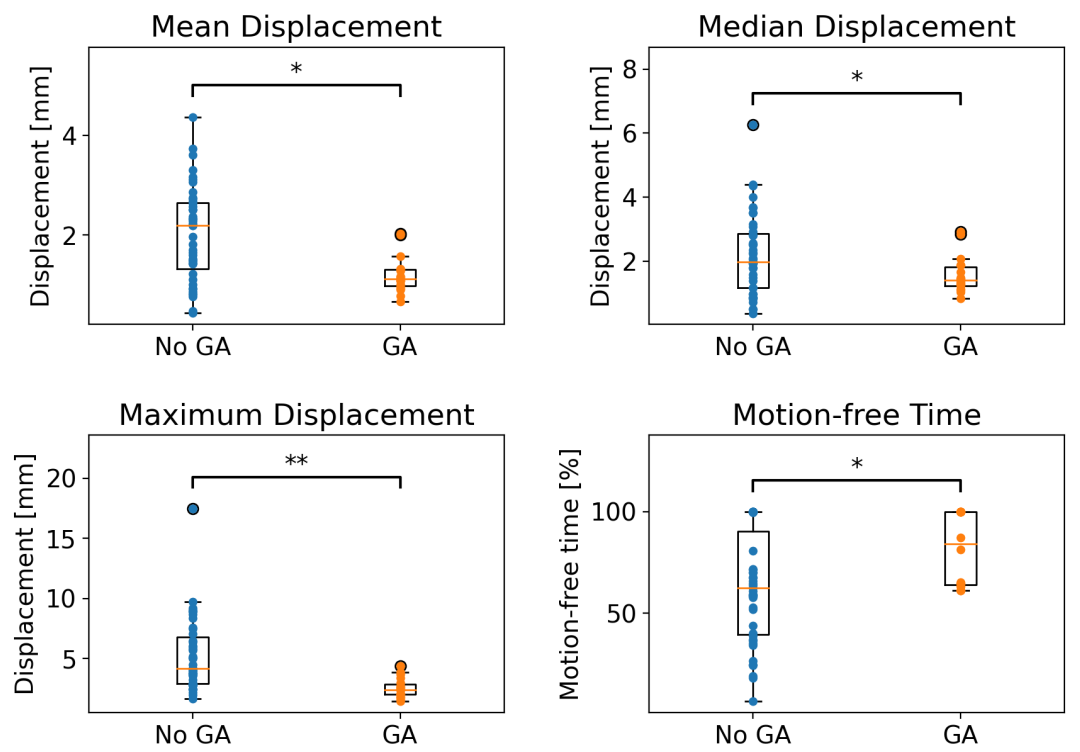

Figure 4: Comparison of motion metrics calculated with point cloud centroid for children with and without GA. Statistical significance after FDR correction is indicated by * $/ * *(p<$ $0.05 / p<0.001)$.

tically significant difference between anaesthetised and awake children. Please note several values corresponding to a substantial amount of motion even for children under anaesthesia.

The metrics for the 16 analysed brain regions can be compared in figures $\mathrm{S.4}$. S.5. S.6 and S.7 of the Supplementary Material. Maximum and mean displacement as well as motion free time vary statistically significantly between patients with and without GA for all regions. Median displacements are distributed over a larger range and only show statistical significance for some regions, but the same trend as for the other metrics is still observable.

\subsection{Decomposition of motion into translation and rotation}

We extracted translational and rotational components for both patient groups and compare them in Figure 5 For both groups, the absolute translational component along the z-axis, which is the axis going into the scanner bore and pointing towards the top of the head, is significantly larger than the absolute 

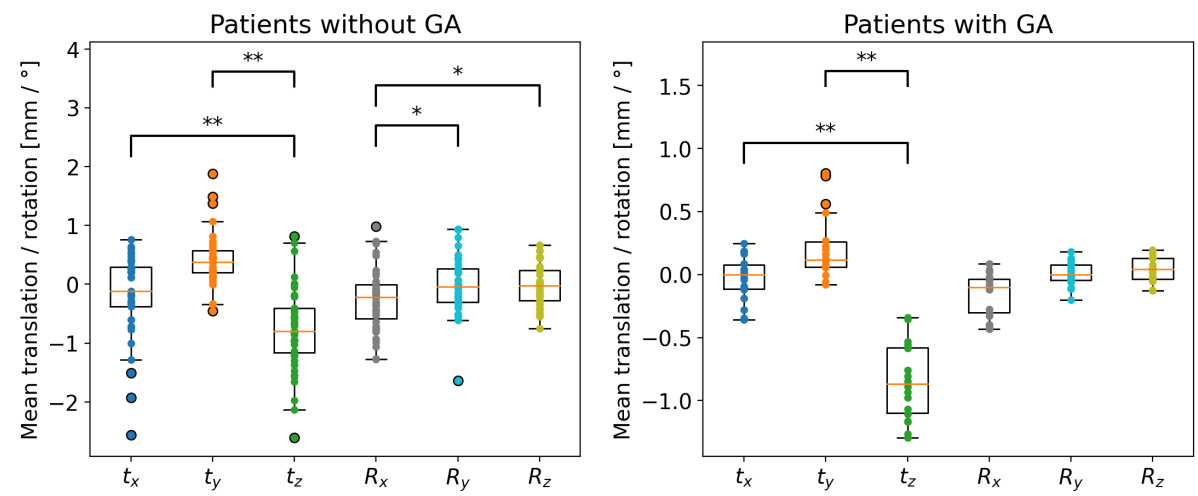

Figure 5: Decomposition of transformation matrices into translational $\left(t_{x}, t_{y}, t_{z}\right)$ and rotational components $\left(R_{x}, R_{y}, R_{z}\right)$, for children without and with GA. Statistical significance after FDR correction is indicated by $* / * *(p<0.05 / p<0.001)$; please note that significance was tested on absolute translations and rotations, whereas mean values of positive and negative components are shown in the graph for visualising the direction of the motion. Notice the different scales for children without and with GA.

values along the $\mathrm{x}$ - and $\mathrm{y}$-axis. Regarding directionality of this translation, both patient groups move in the negative z-direction, i.e. slide downwards out of the scanner. For the children without GA, the absolute rotational component around the $\mathrm{x}$-axis is significantly larger than the components around the other axes, whereas for the GA group no significant differences between the rotations are observed. In the RAS system, the x-axis points from right to left.

\subsection{Motion of different brain regions}

To further analyse the motion patterns, we calculated the median displacement on the $\mathrm{x}-, \mathrm{y}-$ and $\mathrm{z}$-axis for each brain region, respectively. Figure 6 shows the results for 4 cortical and 4 subcortical regions. The results for the remaining 8 regions analysed in this study are available in the Supplementary Material (Figure S.8). The magnitude of the motion on the z-axis exceed that of the other axes for both patient groups, even though the differences are smaller for the group without GA. This difference is statistically significant for both groups and all 16 regions except the comparison of z- and y-axis for the right hemisphere precentral region in the patient group without GA (see Figure S.8). 
Patients without GA, subcortical regions
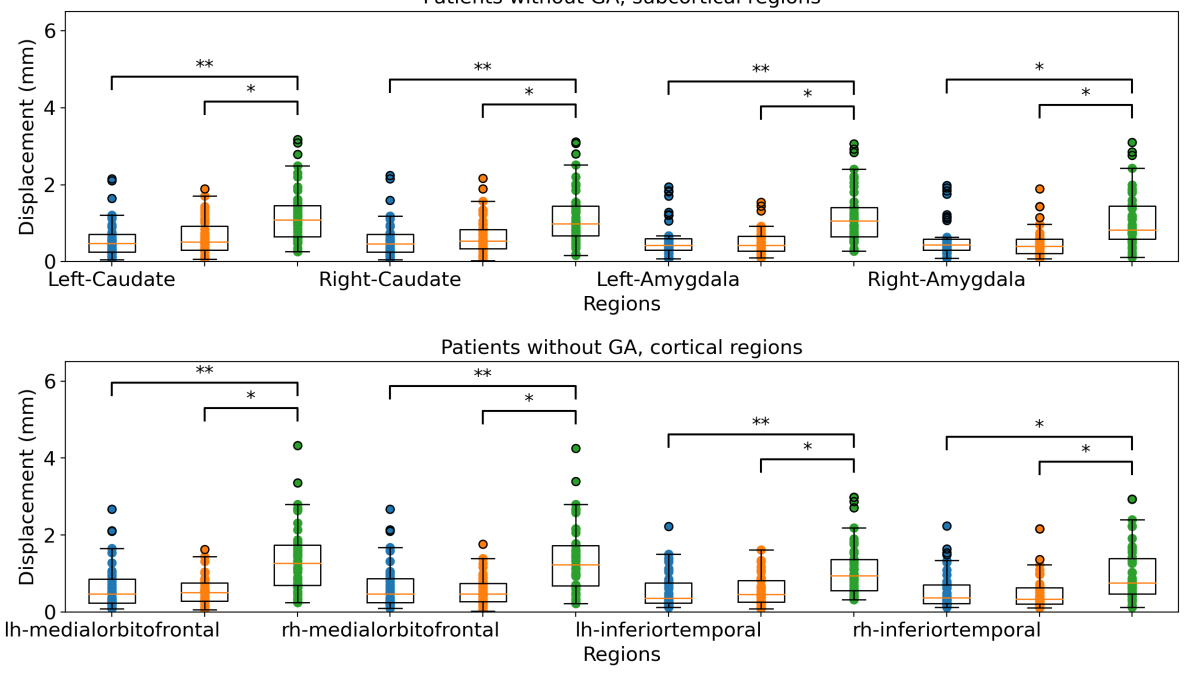

Patients with GA, subcortical regions

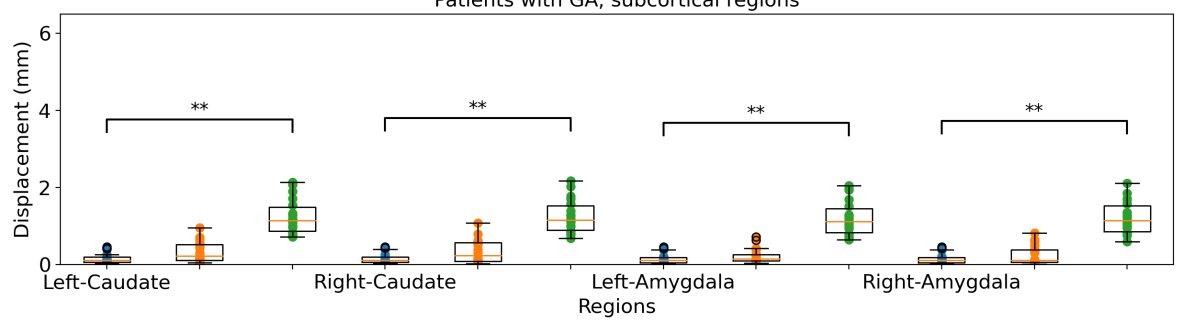

Patients with GA, cortical regions

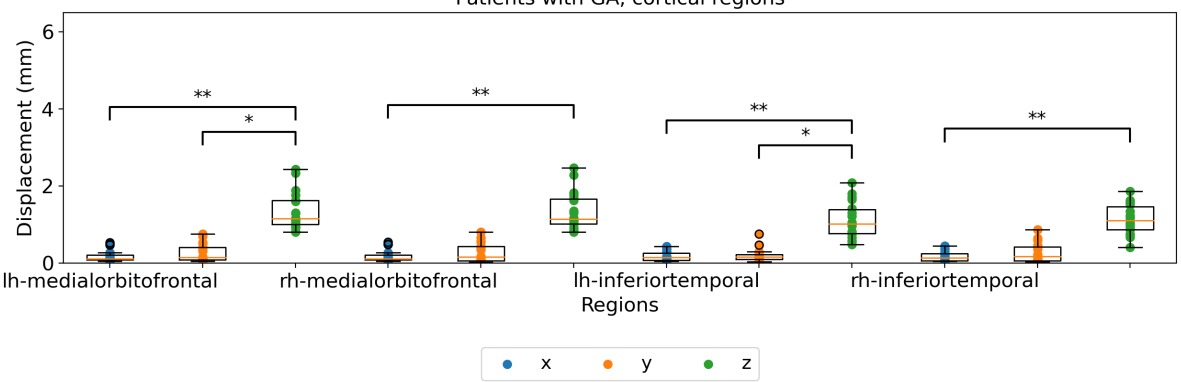

Figure 6: Comparison of median distance on each axis for 4 cortical and 4 subcortical regions split for the $\mathrm{x}-, \mathrm{y}$, and $\mathrm{z}$-axis (blue, orange and green, respectively). The upper two plots show patients without GA, the lower two patients with GA. Statistical significance after FDR correction is indicated by $* / * *(p<0.05 / p<0.001)$. 


\section{Discussion}

We analysed motion data from anaesthetised and awake children during MRI examination using the same protocol and the same MR scanner for all scans, which to our knowledge has not been reported so far.

Comparing motion metrics like mean, median, maximum displacement and motion-free time in Figure 4, confirmed our expectations that children without GA moved significantly more than children with GA for all analysed metrics. Nevertheless, residual movement was still observed for anaesthetised children. As described in the Introduction, Afacan et al. [1] investigated the correlation of motion metrics like mean and maximum displacement and motion-free time with image quality based on motion estimates, which were measured with two sensors placed on the patient's forehead. For enabling comparisons with their work, we calculated our metrics for the point cloud centroid, which corresponds to a point close to the middle of the nose bridge, as visualised in Supplementary Figure S.3. We reported both mean and median displacement, since the displacements over time were not normally distributed, which we confirmed with a Kolmogorow-Smirnow test for normality. Even though Afacan et al. did not find significant differences between radiologic evaluation and maximum displacement, we reported maximum movement since it provides valuable information about the severity of motion spikes, especially in the context of motion correction.

Furthermore, in Figure 4 we showed that even anaesthetised children moved substantially across all metrics: with mean displacements of up to $1.9 \mathrm{~mm}$, maximum displacements of up to $4.4 \mathrm{~mm}$ and motion-free times down to $58 \%$. Afacan et al. [1], who analysed the correlation of motion metrics with radiologic evaluation of image quality, reported motion-free time medians of $38 \% \pm 21$, $74 \% \pm 27,97 \% \pm 17$ and $99 \% \pm 3$ for the grades $1,2,3$, and 4, respectively (4 being the best image quality). Together with our results of motion-free time values down to $58 \%$, this indicates that GA does not guarantee high image quality due to considerable residual motion in several cases. Our different 
threshold for calculating motion-free times, does not falsify these conclusions, since lowering our threshold from $2 \mathrm{~mm}$ to $0.2 \mathrm{~mm}$ (as in [1]) would only decrease our percentages of motion free times. Please note that Afacan et al. also used a different motion tracking device, an electromagnetic tracker [24] developed by Robin Medical Inc. (Baltimore, MD). For other motion tracking devices using external fitted tools, like the Polaris Vicra, inaccuracy in the motion estimates has been shown due to dislocation of the tool relative to the head [25].

In order to obtain more insight into which types of movement children perform most commonly during MR examinations, we decomposed the transformation matrices for each time point into translation and rotation. For both patient groups, we compared the components along / around the three axes. In Figure 5. we showed for both patient groups significantly larger absolute translation along the $\mathrm{z}$-axis compared to $\mathrm{x}$ - and $\mathrm{y}$-axes. For the children without GA, we additionally observed significantly larger rotations around the $\mathrm{x}$-axis compared to the $\mathrm{y}$ - and z-axes. The z-translation, which corresponds to translation along the axis through the scanner bore, can be interpreted as a drift motion, especially for the children with GA. This becomes apparent when looking at the complete motion data throughout the whole scan session e.g. in Figure 3 For most of the GA patients one can observe a continuous increase in the displacement along the z-axis from the beginning until the end of the examination. Since we observed negative z-translation values for both patient groups, this motion corresponds to a gliding downwards out of the scanner. The rotational component around the $\mathrm{x}$-axis observed for children without GA corresponds to a nodding motion, which together with a sliding in negative z-direction has previously also been observed as primary motion for adults in the MR scanner [18, 26]. For children specifically, this movement can be explained by parents or a screen with a movie being positioned at the end of the scanner bore. Thus, our analysis provides important conclusions for clinical examinations of children, namely that avoiding nodding motion should play a larger role in training children before an examination.

Finally, we analysed how the children's motion affects different parts of the 
brain. For this, we chose 8 cortical and 8 subcortical regions of the brain, including paired regions from the left and right side of the brain. This allowed us to compare the influence of motion on the left and right side as well as back, front and central parts of the brain. We calculated the displacement along each axis for the selected regions. All in all, this analysis is in accordance with the results from the matrix decomposition, i.e. that the largest displacement was observed for the z-axis (cf. Figure6). For the GA group very little motion was observed on the $\mathrm{x}$ - and $\mathrm{y}$-axes. The group without GA showed more motion on the $\mathrm{x}$ - and $\mathrm{y}$-axes, but the z-axis was still dominating. The differences were statistically significant for all regions, except the precentral region of the right hemisphere for the patient group wihout GA. For this region, the median motion was more distributed across y- and z-axes.

Our study is not without limitations. First, it is limited by the unbalanced distribution between the groups with and without GA. A larger GA group might enable higher statistical significance for comparing the motion metrics between both groups. Another potential source of error is the fact that FreeSurfer is optimised for adult brain anatomy, which could lead to slightly wrong segmentation of the brain regions. However, each segmentation was verified manually. Lastly, we excluded approximately one third of the data set, amongst other things due 240 to poor scan quality impeding a successful segmentation by FreeSurfer. This could lead to excluding scans with large amounts of motion and thus, underestimating the true motion of children in general. However, our comparison of included and excluded scans in Supplementary Figure S.2 confirmed that excluded scans did not systematically have larger amounts of motion, apart from two outlier scans (see Figure S.2.

\section{Conclusion}

Considering the presented results together with the discussed limitations, our study puts some light onto how children move during a (PET/)MR scan, when they are awake as well as anaesthetised. First of all, we showed that even 
children under GA show substantial amount of motion, which strengthens the need for alternative methods such as motion correction techniques and adequate preparation of the children to avoid motion artefacts in case the motion causes clinically image degrading artefacts. For the anaesthetised children, the clinical evaluation of image quality reported in this study shows no problem with motion artefacts; free motion, however, requires motion correction to avoid GA for more children. In addition, our data indicates that clinical routines as well as training methods under development should be given special attention to prevent nodding motion. Similarly, the higher prevalence of nodding motion and translation along the z-axis should be taken into account when optimizing and testing motion correction methods. In order to limit motion artefacts further, the application of MR sequences that are more robust towards these two types of motion regarding slicing and phase encoding direction should be considered.

\section{Acknowledgment}

We highly appreciate the scanner assistance of technologists Karin Stahr and Marianne Federspiel, and of radiographers Jákup Martin Poulsen and Nadia Azizi. This work was supported by The Danish Childhood Cancer Foundation (2014-34, 2015-48), as well as the Elsass Fonden (18-3-0147) and an unrestricted grant from the Novo Nordisk and Novozymes Talent Program.

\section{Data and Code availability statement}

270 Due to regulations according to the Danish Data Protection Agency, the very sensitive patient data including the MRI sequences are not available.

The code used to preprocess and create the results used in this manuscript mostly consists of open-source software (FreeSurfer and Python). Code for running the analysis can be found at GitHub. 
[1] O. Afacan, B. Erem, D. P. Roby, N. Roth, A. Roth, S. P. Prabhu, S. K. Warfield, Evaluation of motion and its effect on brain magnetic resonance image quality in children, Pediatric Radiology 46 (12) (2016) 1728-1735. doi:10.1007/s00247-016-3677-9.

[2] F. Godenschweger, U. Kägebein, D. Stucht, U. Yarach, A. Sciarra, R. Yakupov, F. Lüsebrink, P. Schulze, O. Speck, Motion correction in MRI of the brain, Physics in Medicine and Biology 61 (5) (2016) R32-R56. doi:10.1088/0031-9155/61/5/R32.

[3] J. B. Andre, B. W. Bresnahan, M. Mossa-Basha, M. N. Hoff, C. Patrick Smith, Y. Anzai, W. A. Cohen, Toward quantifying the prevalence, severity, and cost associated with patient motion during clinical MR examinations,

口 Journal of the American College of Radiology 12 (7) (2015) 689-695. doi: $10.1016 / j \cdot j$ acr.2015.03.007. URL http://dx.doi.org/10.1016/j.jacr.2015.03.007

[4] H. M. De Bie, M. Boersma, M. P. Wattjes, S. Adriaanse, R. J. Vermeulen, K. J. Oostrom, J. Huisman, D. J. Veltman, H. A. Delemarre-Van De Waal, Preparing children with a mock scanner training protocol results in high quality structural and functional MRI scans, European Journal of Pediatrics 169 (9) (2010) 1079-1085. doi:10.1007/s00431-010-1181-z.

295 [5] S. B. Runge, N. L. Christensen, K. Jensen, I. E. Jensen, Children centered a care: Minimizing the need for anesthesia with a multi-faceted concept for MRI in children aged 4-6, European Journal of Radiology 107 (July) (2018) 183-187. doi:10.1016/j.ejrad.2018.08.026. URL https://doi.org/10.1016/j.ejrad.2018.08.026

[6] J. M. Slipsager, S. L. Glimberg, J. Søgaard, R. R. Paulsen, H. H. Johannesen, P. C. Martens, A. Seth, L. Marner, O. M. Henriksen, O. V. Olesen, L. Højgaard, Quantifying the Financial Savings of Motion Correction in 
Brain MRI: A Model-Based Estimate of the Costs Arising From Patient Head Motion and Potential Savings From Implementation of Motion Correction, Journal of Magnetic Resonance Imaging 52 (3) (2020) 731-738. doi:10.1002/jmri.27112.

[7] J. E. Havidich, M. Beach, S. F. Dierdorf, T. Onega, G. Suresh, J. P. Cravero, Preterm versus term children: Analysis of sedation/anesthesia adverse events and longitudinal risk, Pediatrics 137 (3). doi:10.1542/ peds.2015-0463

[8] K. Johnson, A. Page, H. Williams, E. Wassemer, W. Whitehouse, The use of melatonin as an alternative to sedation in uncooperative children undergoing an MRI examination, Clinical Radiology 57 (6) (2002) 502506. doi:10.1053/crad.2001.0923.

[9] E. Törnqvist, A. Månsson, I. Hallström, Children having magnetic resonance imaging: A preparatory storybook and audio/visual media are preferable to anesthesia or deep sedation, Journal of Child Health Care 19 (3) (2015) 359-369. doi:10.1177/1367493513518374.

[10] A. D. Edwards, O. J. Arthurs, Paediatric MRI under sedation: Is it necessary? What is the evidence for the alternatives?, Pediatric Radiology 41 (11) (2011) 1353-1364. doi:10.1007/s00247-011-2147-7.

[11] R. Frost, P. Wighton, F. I. Karahanoğlu, R. L. Robertson, P. E. Grant, B. Fischl, M. D. Tisdall, A. van der Kouwe, Markerless high-frequency prospective motion correction for neuroanatomical MRI, Magnetic Reso325 nance in Medicine 82 (1) (2019) 126-144. doi:10.1002/mrm.27705.

[12] M. D. Tisdall, A. T. Hess, M. Reuter, E. M. Meintjes, B. Fischl, A. J. Van Der Kouwe, Volumetric navigators for prospective motion correction and selective reacquisition in neuroanatomical MRI, Magnetic Resonance in Medicine 68 (2) (2012) 389-399. doi:10.1002/mrm.23228. 
[13] J. Maclaren, M. Herbst, O. Speck, M. Zaitsev, Prospective motion correction in brain imaging: A review, Magnetic Resonance in Medicine 69 (3) (2013) 621-636. doi:10.1002/mrm.24314.

[14] J. M. Slipsager, S. L. Glimberg, D. Gallichan, L. Højgaard, R. R. Paulsen, O. V. Olesen, Clinical Implementation of Fast Markerless Motion Correc335 tion in K-Space of Structural 3D MR-Images of the Brain, in: ISMRM $2019,2019$.

[15] H. Jung, K. Sung, K. S. Nayak, E. Y. Kim, J. C. Ye, K-t FOCUSS: A general compressed sensing framework for high resolution dynamic MRI,

घ Magnetic Resonance in Medicine 61 (1) (2009) 103-116. doi:10.1002/ mrm.21757.

[16] J. Zbontar, F. Knoll, A. Sriram, T. Murrell, Z. Huang, M. J. Muckley, A. Defazio, R. Stern, P. Johnson, M. Bruno, M. Parente, K. J. Geras, J. Katsnelson, H. Chandarana, Z. Zhang, M. Drozdzal, A. Romero, M. Rabbat, P. Vincent, N. Yakubova, J. Pinkerton, D. Wang, E. Owens, C. L. Zitnick, M. P. Recht, D. K. Sodickson, Y. W. Lui, fastMRI: An Open Dataset and Benchmarks for Accelerated MRI (2018) 1-35arXiv:1811.08839.

URL http://arxiv.org/abs/1811.08839

[17] H. Zhang, B. Dong, A Review on Deep Learning in Medical Image ReconstructionlarXiv:1906.10643.

$350 \quad$ URL http://arxiv.org/abs/1906.10643

[18] N. W. Churchill, A. Oder, H. Abdi, F. Tam, W. Lee, C. Thomas, J. E. Ween, S. J. Graham, S. C. Strother, Optimizing preprocessing and analysis pipelines for single-subject fMRI. I. Standard temporal motion and physiological noise correction methods, Human Brain Mapping 33 (3) (2012) 609-627. doi:10.1002/hbm.21238.

[19] L. Marner, K. Nysom, A. Sehested, L. Borgwardt, R. Mathiasen, O. M. Henriksen, M. Lundemann, P. Munck af Rosenschöld, C. Thomsen, 
L. Bøgeskov, J. Skjøth-Rasmussen, M. Juhler, A. Kruse, H. Broholm, D. Scheie, T. Lauritsen, J. L. Forman, P. S. Wehner, L. Højgaard, I. Law, Early postoperative 18f-fet pet/mri for pediatric brain and spinal cord tumors Journal of Nuclear Medicine 60 (8) (2019) 1053-1058. arXiv:https: //jnm.snmjournals.org/content/60/8/1053.full.pdf, doi:10.2967/ jnumed.118.220293.

URL https://jnm.snmjournals .org/content/60/8/1053

[20] B. Fischl, FreeSurfer, NeuroImage 62 (2) (2012) 774-781. doi:10.1016/ j.neuroimage.2012.01.021.

[21] J. M. Slipsager, A. H. Ellegaard, S. L. Glimberg, R. R. Paulsen, M. Dylan Tisdall, P. Wighton, A. Van Der Kouwe, L. Marner, O. M. Henriksen, I. Law, O. V. Olesen, Markerless motion tracking and correction for PET, MRI, and simultaneous PET/MRI, PLoS ONE 14 (4). doi:10.1371/journal.pone.0215524.

[22] O. V. Olesen, M. R. Jørgensen, R. R. Paulsen, L. Højgaard, B. Roed, R. Larsen, Structured Light 3D Tracking System for Measuring Motions in PET Brain Imaging, in: Proceedings of SPIE Medial Imaging - The International Society for Optical Engineering, Vol. 7625, 2010, pp. 121135.

[23] Y. Benjamini, Y. Hochberg, Controlling the false discovery rate: A practical and powerful approach to multiple testing, Journal of the Royal Statistical Society: Series B (Methodological) 57 (1) (1995) 289-300. arXiv:https://rss.onlinelibrary. wiley.com/doi/pdf/10.1111/j.2517-6161.1995.tb02031.x. doi:https://doi.org/10.1111/j.2517-6161.1995.tb02031.x.

1 URL https://rss.onlinelibrary.wiley.com/doi/abs/10.1111/j. 2517-6161.1995.tb02031.x

[24] A. Gholipour, M. Polak, A. van der Kouwe, E. Nevo, S. K. Warfield, a Motion-robust mri through real-time motion tracking and retrospective 
super-resolution volume reconstruction, Annual International Conference of the IEEE Engineering in Medicine and Biology Society. IEEE Engineering in Medicine and Biology Society. Annual International Conference 2011 (2011) 5722-5725. doi:10.1109/iembs.2011.6091385.

URL https : //europepmc .org/articles/PMC3687083

[25] S. H. Keller, M. Sibomana, O. V. Olesen, C. Svarer, S. Holm, F. L. Andersen, L. Højgaard, Methods for motion correction evaluation using 18f-fdg human brain scans on a high-resolution pet scanner, Journal of Nuclear MedicinearXiv:https://jnm.snmjournals. org/content/early/2012/02/13/jnumed.111.095240.full.pdf doi:10.2967/jnumed.111.095240

11 URL https://jnm.snmjournals.org/content/early/2012/02/13/ jnumed.111.095240

[26] S. H. Keller, C. Hansen, C. Hansen, F. L. Andersen, C. Ladefoged, C. Svarer, A. Kjær, L. Højgaard, I. Law, O. M. Henriksen, A. E. Hansen,

घ Motion correction in simultaneous PET/MR brain imaging using sparsely sampled MR navigators: a clinically feasible tool, EJNMMI Physics 2 (1) (2015) 1-7. doi:10.1186/s40658-015-0118-z. URL http://dx.doi.org/10.1186/s40658-015-0118-z

\section{Supplementary Material}



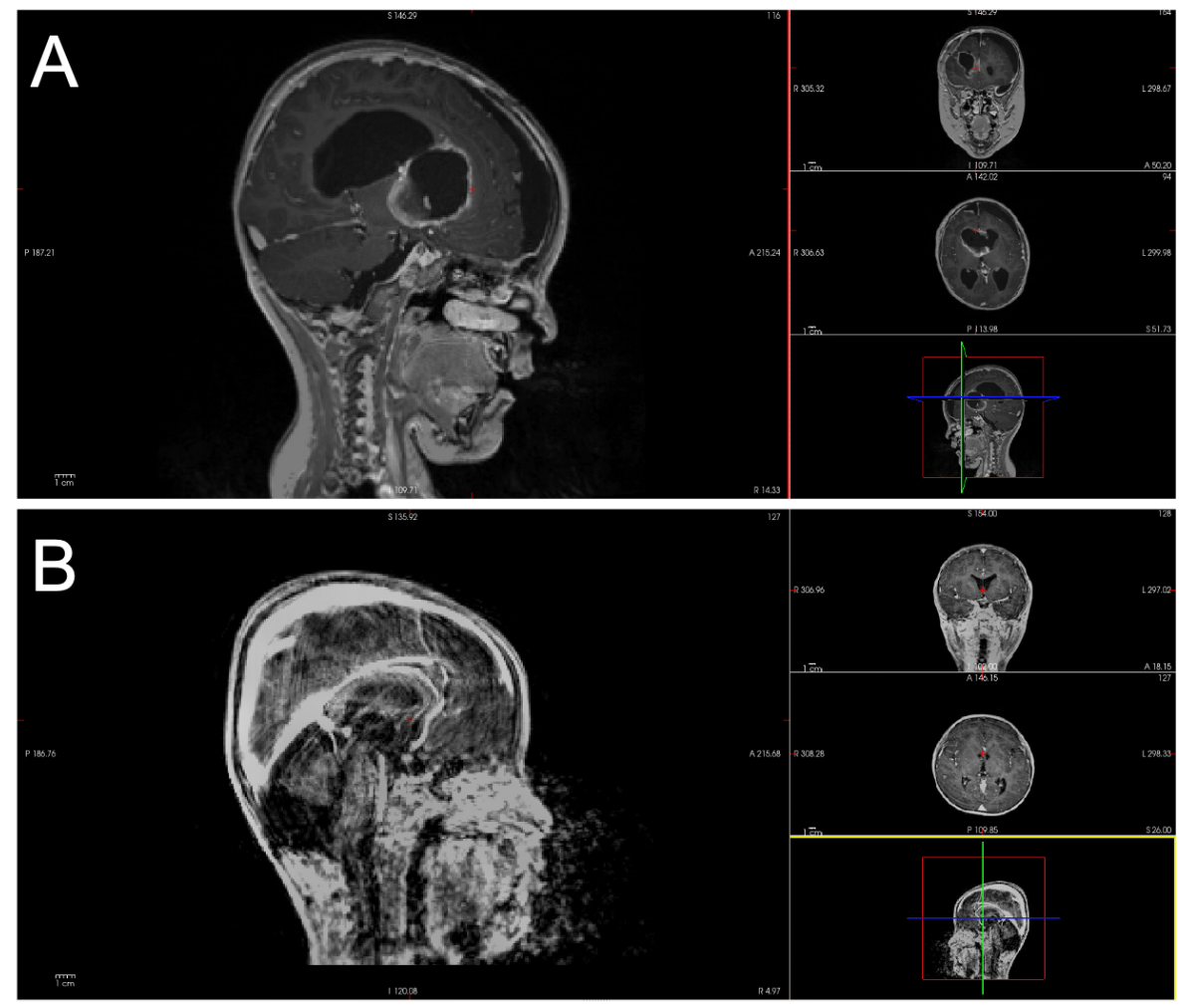

Figure S.1: Examples of excluded scans due to (A) large defects and (B) poor scan quality.

Table S.1: Parameters of the MR sequences describing the type of acquisition, flip angle, repetition, echo as well as inversion time and voxel size.

\begin{tabular}{c|c|c|c|c} 
Sequence & Acquisition type & Flip angle $\left[^{\circ}\right]$ & TR/TE $/$ TI $[\mathbf{m s}]$ & Voxel size $[\mathbf{m m}]$ \\
\hline $\mathrm{T}_{1}$-weighted MPRAGE & $3 \mathrm{D}$ & 9 & $1900 / 2.52 / 900$ & $1.00 \times 1.00 \times 1.00$ \\
$\mathrm{~T}_{1}$-weighted STIR & $2 \mathrm{D}$ & 150 & $2000 / 34 / 800$ & $0.45 \times 0.45 \times 4.00$ \\
$\mathrm{~T}_{2}$-weighted FLAIR (transversal) & $2 \mathrm{D}$ & 150 & $9000 / 85 / 2500$ & $0.69 \times 0.69 \times 4.00$ \\
$\mathrm{~T}_{2}$-weighted FLAIR (coronal) & $2 \mathrm{D}$ & 130 & $9000 / 95 / 2500$ & $0.43 \times 0.43 \times 4.00$ \\
$\mathrm{~T}_{2}$-weighted Blade & $2 \mathrm{D}$ & 140 & $4000 / 118 /-$ & $0.72 \times 0.72 \times 5.00$
\end{tabular}



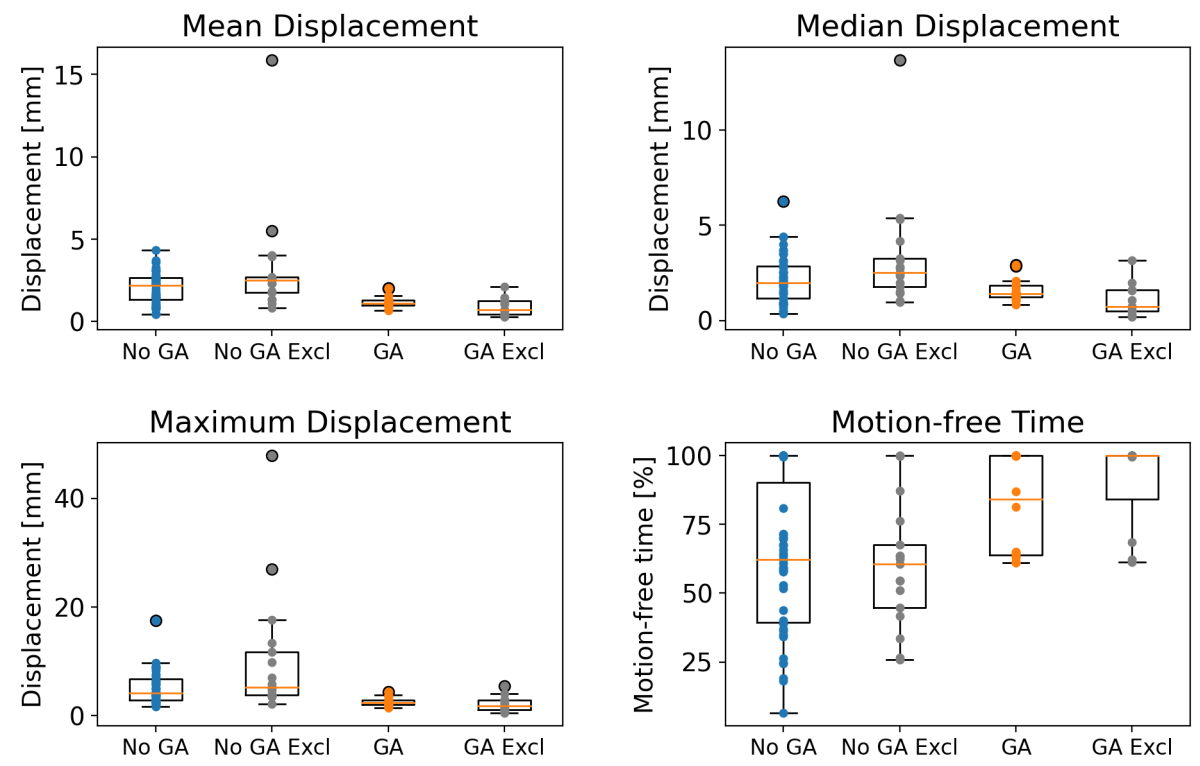

Figure S.2: Motion metrics for point centroid of scans excluded due to poor image quality compared to included scans. The analysis of the excluded scans is performed analogously to the analysis shown in Figure 4 Except two outlier scans with significantly larger motion in the group without GA, the excluded patients show similar amount of motion as the included patients. 


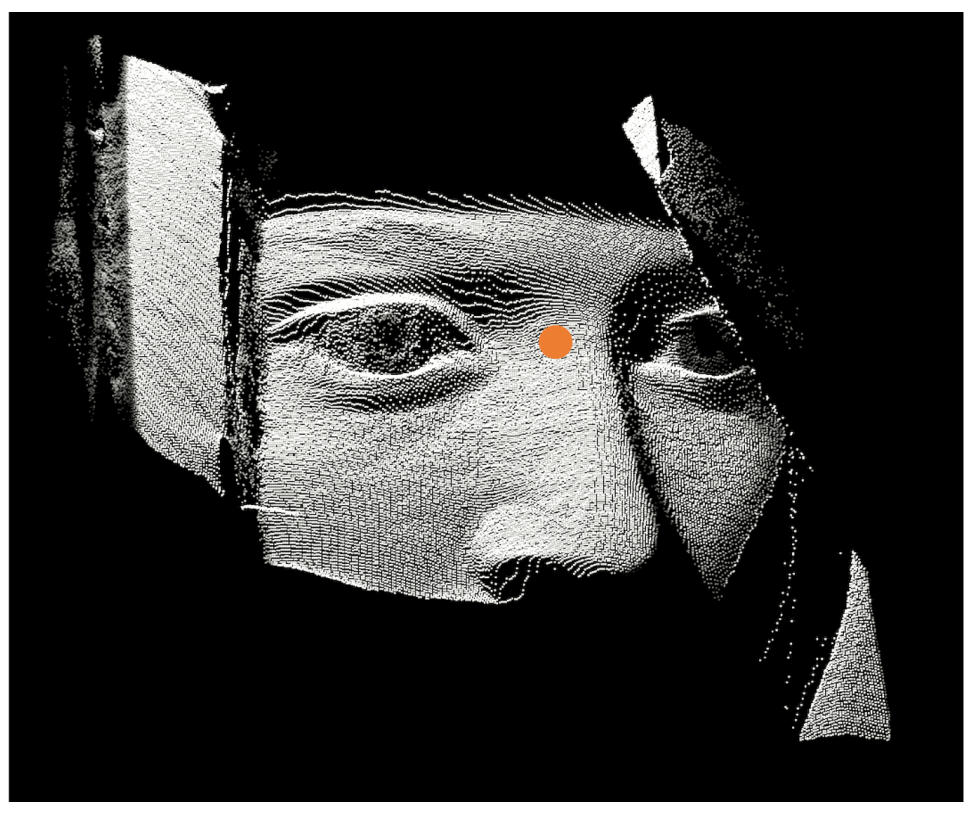

Figure S.3: Example of the Tracoline point cloud for an adult. The point cloud centroid is schematically visualised by the orange circle. Please note that the field of view varies with head size and curvature of the face; for children a larger part of the face might be covered.
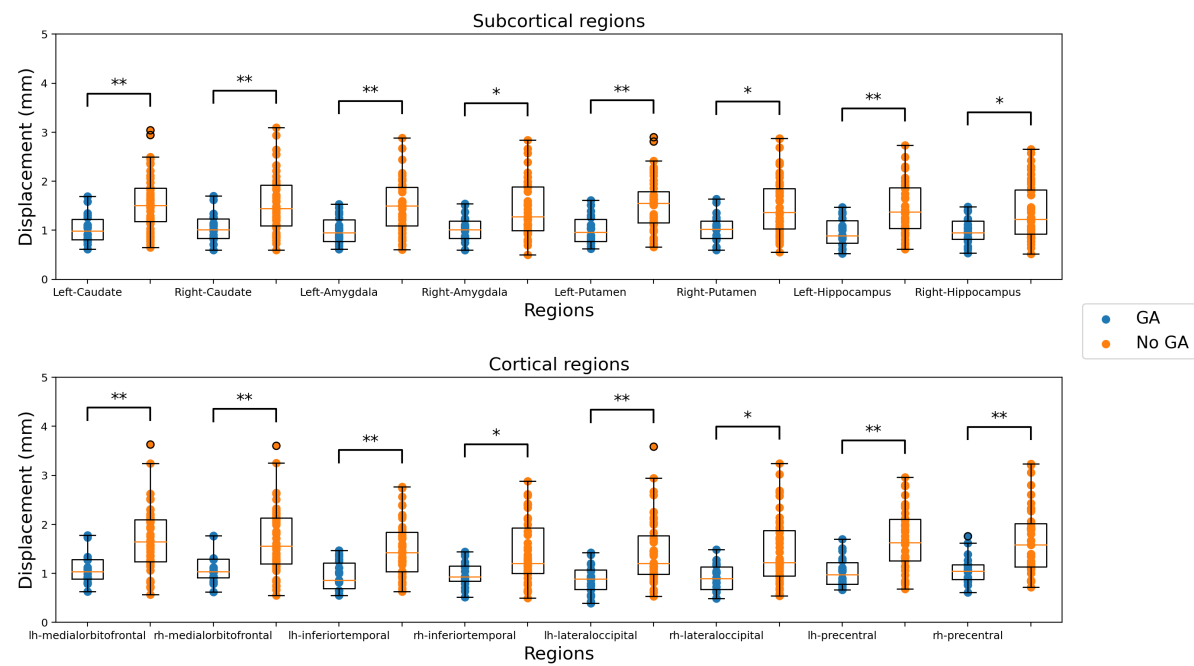

Figure S.4: Comparison of mean displacement for all cortical and subcortical regions. Statistical significant differences after Benjamini-Hochberg correction are indicated by */** $(p<0.05 / p<0.001)$. 

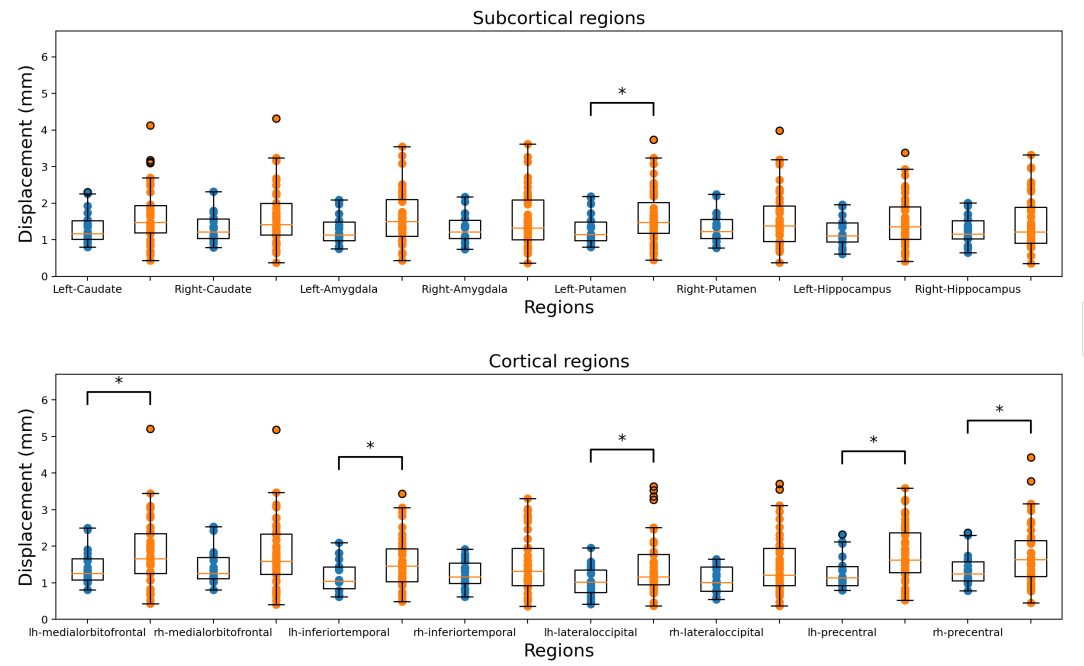

Figure S.5: Comparison of median displacement for all cortical and subcortical regions. Statistical significant differences after Benjamini-Hochberg correction are indicated by $*(p<0.05)$.
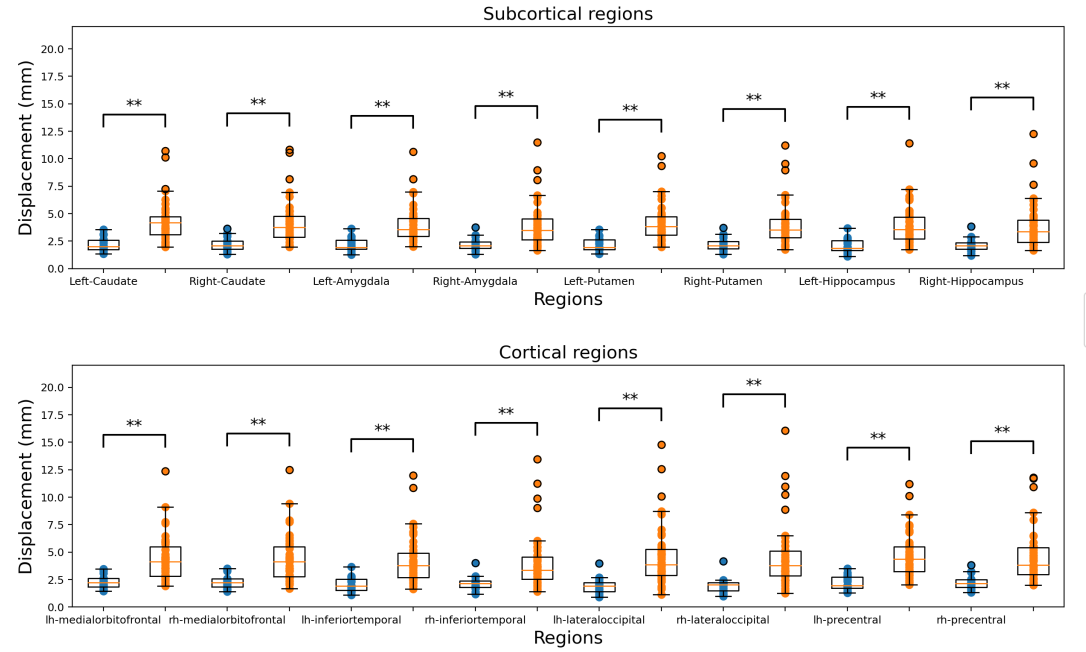

Figure S.6: Comparison of maximum displacement for all cortical and subcortical regions. Statistical significance after Benjamini-Hochberg correction is indicated by $* *(p<0.001)$. 

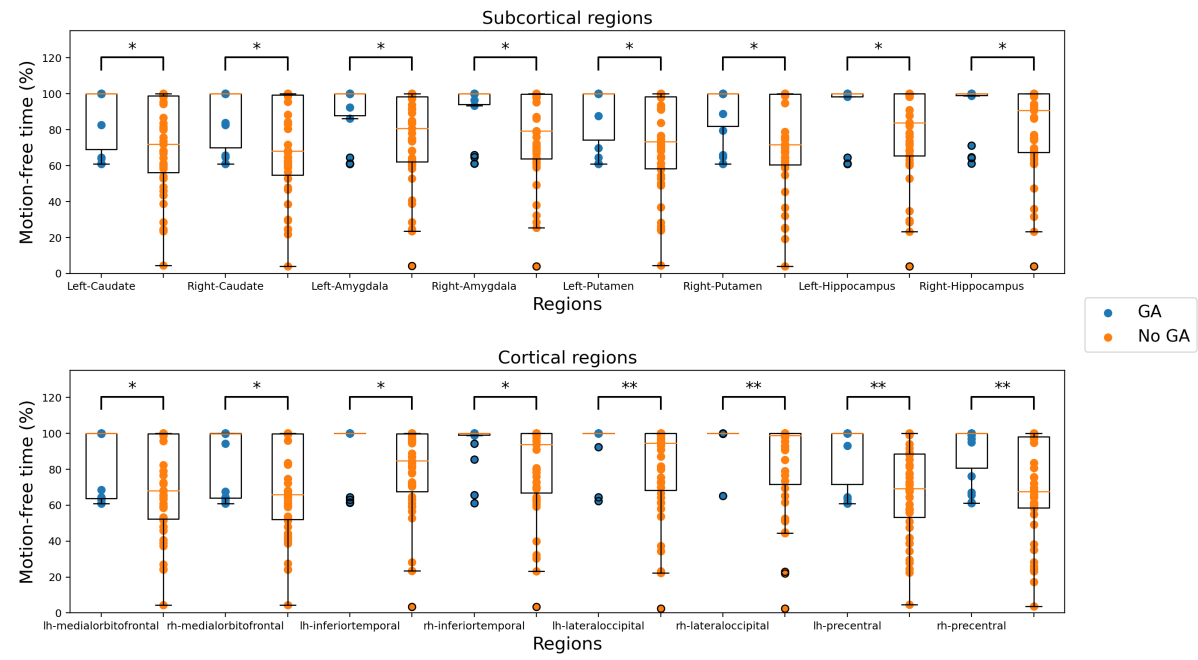

Figure S.7: Comparison of motion-free time for all cortical and subcortical regions. Statistical significance after Benjamini-Hochberg correction is indicated by $* / * *(p<0.05 / p<0.001)$. 


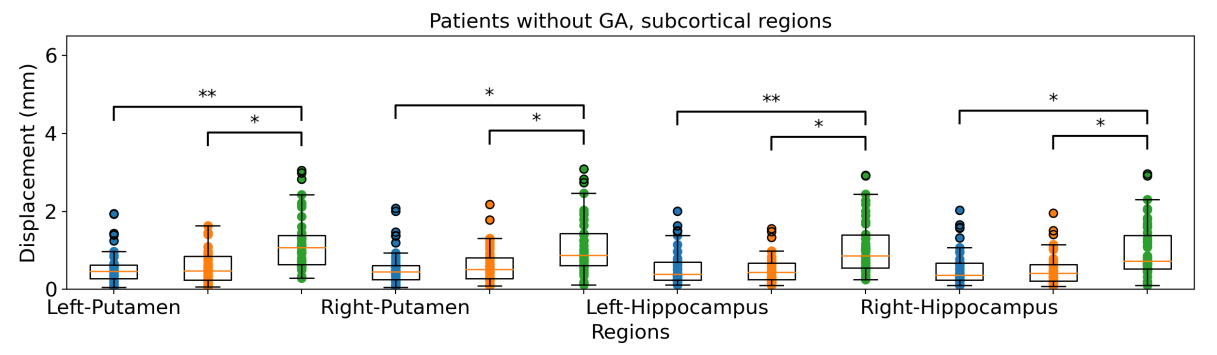

Patients without GA, cortical regions
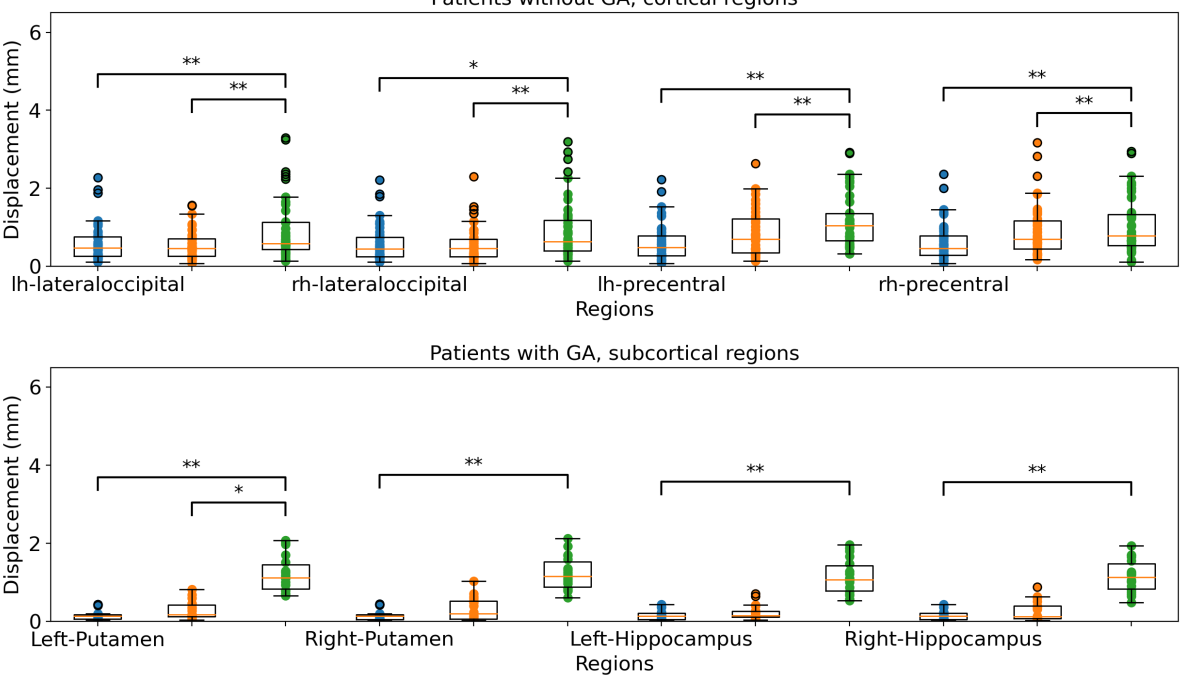

Patients with GA, cortical regions

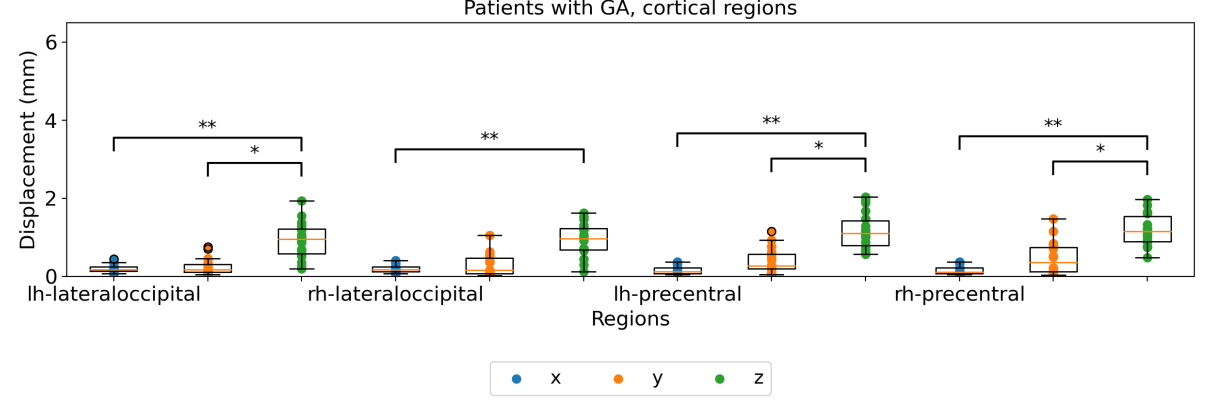

Figure S.8: Comparison of median distance on each axis for the 4 cortical and 4 subcortical regions not included in Figure 6 The top two plots show patients without GA, the lower two patients with GA. Statistical significance after Benjamini-Hochberg correction is indicated by $* / * *(p<0.05 / p<0.001)$. 